\title{
Cyclin-dependent Kinase 9 Induces Regional and Global Genomic DNA Methylation Via Influencing DNMT Gene Expression in Mouse Myoblast C2C12 Cells During Differentiation
}

\author{
Leila Abkhooie ${ }^{1,2,3}$, Mostafa Moradi Sarabi ${ }^{4,1^{*}}$, Houman Kahroba ${ }^{1,2}$, Hossein Ghanbarian ${ }^{5}$, Soheila Montazer \\ Saheb², Vahideh Tarhriz $^{2,1^{*}}$, Mohammad Saeid Hejazi ${ }^{1,2,6}$
}

\begin{abstract}
Objectives: Cyclin-dependent kinases (CDKs) including Cdk9 have been associated with cardiac differentiation. The increasing evidence has proposed that $C d k 9$ overexpression can regulate the epigenome. However, the current research is the first report of the Cdk9 affection on the regional and global DNA methylation during differentiation.

Materials and Methods: This study examined the effects of $C d k 9$ overexpression on the regional methylation patterns of cardiac miRNAs (miR-1, -133, -206) and myogenic regulatory factors (i.e., MyoD and Myogenin) and promoter DNA methylation in mouse myoblast $\mathrm{C} 2 \mathrm{C} 12$ cells during differentiation by the methylation-specific polymerase chain reaction (MSP-PCR) method. Moreover, the mRNA expression levels of DNMT1, DNMT3A, DNMT3B, and global 5-methyl cytosine (5-mC) levels in mouse myoblast C2C12 cells were quantified during differentiation by RT-qPCR and ELISA methods, respectively.

Results: The results demonstrated that Cdk9 overexpression results in DNA methylation changes in mouse myoblast C2C12 cells. It was found that the average expression levels of DNMTs in line with global DNA methylation significantly increased in Cdk9 transfected cells upon $C d k 9$ overexpression $(P<0.05)$. In addition, the results showed that the regional promoter methylation of miR-1 and miR-133 genes increased in transfected cells during differentiation. An interesting possibility raised by our study is that further active global DNA methylation observed in Cdk9-transfected C2C12 cells can be clarified through the increased DNMT expression by $C d k 9$ in these cells.

Conclusions: In general, our study provides a comprehensive mechanism that $C d k 9$ can promote epigenetic changes and modulate global and regional DNA methylation profiling of myoblast cells during differentiation.

Keywords: MicroRNA, Cdk9, DNA methylation, Gene expression, Myoblast cell differentiation
\end{abstract}

\begin{abstract}
Introduction
Epigenetics is a set of multiple processes containing DNA methylation, alterations in histone structures, and the affection of various non-coding RNAs in the expression of genes and chromatin remodeling in the cell $(1,2)$. After the egg cell formation, its development depends on the type of epigenetic changes that occur on the inherited material of the cell (1). DNA methylation is an epigenetic modification that has a critical role in different molecular pathways including chromatin remodeling, gene silencing, inactivation of X-chromosome, and genomic imprinting (3). DNA methylation occurs within CpG dinucleotide pairs on the 5 ' position of cytosine bases, followed by 5 -methylcytosine (5-mC) formation (4). Approximately $70 \%$ of annotated mammalian promoters belong to
\end{abstract}

CpG islands so that its methylation is essential for the expression and development of genes, as well as genome stability $(5,6)$. DNMT1, DNMT3A, and DNMT3B are activated forms that are responsible for DNA methylation. The main role of DNMT1 is the methylation of DNA in stable forms for the transmission of methylation patterns to daughter cells during cell divisions. DNMT3A and $D N M T 3 B$ function as de novo DNMT enzymes and establish the patterns of genomic methylation in the post-fertilization stage (7). Additionally, all three DNMTs are needed for fetal development, and any dysfunction in this enzymatic system has profound effects on the developmental process and disease (1). According to (8-10), muscle differentiation as a process with multiple steps is controlled by myogenic regulatory factors (MRFs)

Received 4 December 2020, Accepted 7 February 2021, Available online 19 March 2021

'Department of Molecular Medicine, Faculty of Advanced Medical Sciences, Tabriz University of Medical Sciences, Tabriz, Iran. ${ }^{2}$ Molecular Medicine Research Center, Biomedicine Institute, Tabriz University of Medical Sciences, Tabriz, Iran. ${ }^{3}$ Department of Medical Biotechnology, Faculty of Medicine, Lorestan University of Medical Sciences, Khorramabad, Iran. ${ }^{4}$ Department of Clinical Laboratory Sciences, School of Allied Medicine, Lorestan University of Medical Sciences, Khorramabad, Iran. ${ }^{5}$ Cellular and Molecular Biology Research Center, Shahid Beheshti University of Medical Sciences, Tehran, Iran. ${ }^{6}$ Department of Pharmaceutical Biotechnology, Faculty of Pharmacy, Tabriz University of Medical Sciences, Tabriz, Iran.

*Corresponding Authors: Mostafa Moradi Sarabi, Tel: +98 -663342 0030, Email: sarabless2003@yahoo.com; Vahideh Tarhriz, Tel: +98 -9142575057, Email: tarhrizv@tbzmed.ac.ir 


\section{Key Messages}

- Cyclin-dependent kinase $9(\mathrm{Cdk} 9)$ is a triggering factor for the induction of differentiation in the primary stage of myogenesis. Our finding showed that $C d k 9$ can promote epigenetic changes via increasing of DNMTs expression levels and modulation of global DNA methylation profiling in myoblast $\mathrm{C} 2 \mathrm{C} 12$ cell line during differentiation.

and microRNAs (miRNAs). For appropriate cardiac development, the expression of these MRFs including Myogenin and MyoD (myoblast determination protein) is critical for various stages of myogenesis and myogenic processes (11). Although MyoD, Myf5, and Myf6 are responsible for muscle formation, the functional role of Myogenin is differentiation and accompanied by $M y o D$, and Myf6 strongly applies a controlling role for the differentiation of myoblasts into adult muscle fibers (12). The modulation of MyoD phosphorylation by Cyclindependent kinase $9(C d k 9)$ is a triggering factor for the induction of cardiac differentiation in the primary stage of cardiogenesis $(13,14)$. In our previous study, we have identified Cdk9 functions on myogenic transcriptional factor and myomiRs in myoblast $\mathrm{C} 2 \mathrm{C} 12$ cells (15). Concerning Cdk9 regulatory roles, it was shown that the constant overexpression of Cdk9 can affect mouse myoblast $\mathrm{C} 2 \mathrm{C} 12$ cell line differentiation. Accordingly, Cdk9 was over-expressed in mouse myoblast $\mathrm{C} 2 \mathrm{C} 12$ cells and the expression level of myogenic transcription factors was determined by qPCR and western blotting. In addition, the expression profile of three main myomiRs including miR-1, miR-133, and miR-206 was investigated during cardiac differentiation. Although it induces cardiac differentiation in the primary stage of cardiomyogenesis, persistent $C d k 9$ overexpression represses differentiation by the alteration of myomiRs and myogenic gene expression. Based on our funding, the transient induction of $C d k 9$ in the primary steps of differentiation is crucial for myogenesis (16). However, the precise mechanism by which $C d k 9$ upregulates these miRNAs in myoblast cells is not understood thoroughly. There is no significant report on whether $C d k 9$ overexpression can affect DNMT gene expression and the promoter methylation of miRNAs and MRFs. Thus, it was hypothesized that $C d k 9$ can affect miRNAs and $M R F$ gene expression by enhancing their DNA methylation. For this purpose, the study focused on evaluating the potential regulatory functions of $C d k 9$ on the promoter DNA methylation status of miR-1, miR206, miR-133, myoD, myogenin, and the gene expression level of DNMTs, as well as genomic DNA methylation in myoblast $\mathrm{C} 2 \mathrm{C} 12$ stem cells.

\section{Materials and Methods}

Cell Line and Cell Culture

Mouse myoblasts $\mathrm{C} 2 \mathrm{C} 12$ cells were purchased from the
Cell Bank of Iran (NCBI, Pasteur Institute, Tehran). All chemicals were purchased from Sigma Aldrich (Munich, Germany) and all reagents were obtained from Gibco Life Technologies (UK). The cell line was grown in the DMEM medium supplemented with $10 \%$ fetal bovine serum (FBS), $2 \mathrm{mM}$ glutamine, streptomycin (and $0.1 \mathrm{mg} /$ $\mathrm{mL}$ ), and penicillin $(100 \mathrm{U} / \mathrm{mL})$ in a humidified $5 \% \mathrm{CO}_{2}$ atmosphere at $37^{\circ} \mathrm{C}$. For inducing muscle differentiation, the mentioned medium was changed with a high-glucose (4.5 g/L) DMEM medium containing 2\% horse serum (17).

\section{Transfection of C2C12 Myoblast With pCEP4/cdk9 Plasmid}

The pCEP4/Cdk9 plasmid has been constructed and transfected for C $C k 9$ overexpression as described in our previous study (16). Briefly, the exponential method was used for electroporation by the Gene Pulser Xcell Systems (Hercules, California) in the DMEM medium. Then, the cells were immediately re-cultured in the DMEM medium with $20 \%$ FBS. The medium was changed with the fresh DMEM medium including $10 \%$ FBS with $300 \mathrm{mg} / \mathrm{mL}$ of hygromycin after 24 hours for the separation of stably transfected cells. The differentiation of the cells toward myocells was carried out by the inclusion of $2 \%$ horse serum in the medium.

\section{Extraction of RNA and RT-qPCR}

Once the stably transfected cells were obtained and proliferated, total RNA was extracted from both nontransfected and transfected cells on days $0,1,2,3$, and 5 after the induction of differentiation using the TriPure isolation reagent (Roche Applied Science, Germany) based on the protocol of the manufacturer. CDNAs were synthesized in $20 \mu \mathrm{L}$ reaction volumes from $2 \mu \mathrm{g}$ of total RNA based on the manufacturer's instruction.

The transcript levels of DNMTs were analyzed by RTqPCR assay and performed three times by using Rotor gene 6000. The sequences of primers are listed in Table 1 . The PCR amplification was performed in $25 \mu \mathrm{L}$ final reaction volume according to the method described by Tarhriz et al (16). The cDNA amplification reactions were carried out as precycling heat treatment at $95^{\circ} \mathrm{C}$ (5 minutes), followed by 35 amplification cycles consisting of denaturation at $95^{\circ} \mathrm{C}$ for 30 seconds, annealing, and extension at $60^{\circ} \mathrm{C}$ for 1 minute. The mRNA level of GAPDH was utilized as the internal control, and the relative quantification of the mRNAs of the samples was calculated using the $2^{-\Delta \Delta C t}$ standard method (18).

Genomic DNA Preparation and Methylation Specific Polymerase Chain Reaction

Genomic DNA was extracted from non-transfected (control) and transfected $\mathrm{C} 2 \mathrm{C} 12$ cells according to the standard method by phenol-chloroform extraction, ethanol precipitation, and digestion with proteinase 
Table 1. Applied Primer Sequences for qRT-PCR

\begin{tabular}{|c|c|c|c|c|}
\hline Gene & Forward Primer & Reverse Primer & $\begin{array}{l}\text { Annealing } \\
\mathrm{T}\left({ }^{\circ} \mathrm{C}\right)\end{array}$ & Product Size (bp) \\
\hline DNMT1 & 5'-ACGGAAACCCAAGGAAGAGTC-3' & 5'-CAGCACCACTCTCTGTGTCTA-3' & 60 & 107 \\
\hline DNMT3A & 5'-ACGGCAGAATAGCCAAGTTCA-3' & 5'-GACGTCTGTGTAGTGGACGG-3' & 60 & 170 \\
\hline DNMT3B & 5'-GGGCCCGGTACTTCTGGGGT -3' & 5'-GGCAGTCCTGCAGCTCGAGC -3' & 60 & 87 \\
\hline GAPDH & 5'-TGATGACATCAAGAAGGTGGTG -3' & 5'-TCCTTGGAGGCCATGTAGGCCAT-3' & 60 & 240 \\
\hline
\end{tabular}

Note. qRT-PCR: Quantitative real-time polymerase chain reaction.

K. The status of the promoter methylation of $M y o D$, Myogenin, and cardiac-specific miRNAs including miR1, miR-206, and miR-133 in C2C12 cells was specified by the methylation-specific polymerase chain reaction (MSP-PCR) method according to the protocol described by Moradi et al $(19,20)$. In brief, $2 \mu \mathrm{g}$ of the genomic DNA from $\mathrm{C} 2 \mathrm{C} 12$ cells was modified with sodium bisulfite. The PCR amplification was carried out using specific primers for the promoter region of MyoD and Myogenin, miR-1, miR-133, and miR-206 in both methylated and unmethylated forms on days $0,1,2,3,4$, and 5. Primers were designed using MethPrimer software (http://www. urogene.org/cgibin/methprimer/methprimer.cgi). In all bisulfite modification reactions, the unmethylated (negative) control was DNA from normal leukocytes, and methylated (positive) control was universal human methylated DNA standards from ZYMO Research (Freiburg, Germany). Table 2 presents the primers and PCR conditions for MSP analysis. The PCR products were evaluated by $1.5 \%$ agarose gel electrophoresis in TAE buffer at a $\mathrm{pH}$ level of 8.0 (44.5 mM Tris-base, $50 \mathrm{mM}$ EDTA). The gels were stained with ethidium bromide (1 g.mL-1) and visualized under UV light.

\section{Global DNA Methylation Analysis}

Genomic DNA methylation in the isolated DNA from non-transfected and transfected $\mathrm{C} 2 \mathrm{C} 12$ cells on days 0,1 , $2,3,4$, and 5 after differentiation induction was tests by the measurement of 5-methylatedcytosines (5-mC) using the ELISA assay kit (Zymo Research, Germany) according to the protocol of the manufacturer. The amount of 5-mC was proportional to the optical density intensity measured in an ELISA plate reader at $450 \mathrm{~nm}$. All measurements were carried out thrice. More details were found in our previously published study (21).

\section{Statistical Analysis}

The obtained data were statistically analyzed using GraphPad Prism and (SPSS, Inc., Chicago). In addition, the mean $( \pm S D)$ was calculated by one-way ANOVA, followed by Tukey's multiple comparison tests. Pearson correlation test was utilized for the analysis of the correlation between DNMT expression and global DNA methylation. $P<0.05$ was considered as the level of statistical significance.

\section{Results}

DNMT1, DNMT3A, and DNMT3B Gene Expression in C2C12 Cells

This study evaluated the expression level of the DNMTs in both non-transfected and transfected cells at days $0,1,2$, 3,4 , and 5 of the $\mathrm{C} 2 \mathrm{C} 12$ cell line after treatment with horse serum for the investigation of the relationship between DNMT expression profiles with DNA methylation changes during the differentiation of $\mathrm{C} 2 \mathrm{C} 12$ cells. The relative expression levels of the DNMTs in both non-transfected and transfected cells (with PCEP4/Cdk9 plasmid) are shown in Figures 1 and 2. The average expression levels of DNMT mRNA were normalized with the expression levels of GAPDH mRNA in both non-transfected and transfected cells at days $0,1,2,3,4$, and 5 of the C2C12 cell line. Non-transfected and Cdk9-transfected $\mathrm{C} 2 \mathrm{C} 12$ cells at days 0 were utilized as a reference, and the expression levels of DNMTs in these cells were adjusted to 1.0, and the expressions in all other cells at days 1-5 were expressed

Table 2. Applied Primer Sequences and Annealing Temperature for Methylation-specific PCR

\begin{tabular}{|c|c|c|c|c|}
\hline Gene & Forward Primer & Reverse Primer & $\begin{array}{l}\text { Annealing } \\
\mathrm{T}\left({ }^{\circ} \mathrm{C}\right)\end{array}$ & $\begin{array}{l}\text { Product } \\
\text { Size (bp) } \\
\end{array}$ \\
\hline \multirow{2}{*}{ Myo D } & U: 5'- GGGTATTATTGGGTGGGTTG -3' & 5'- ACСTTAAAGAACTACTAATTCAA -3' & 54 & 186 \\
\hline & M: 5'- GGTATTATTAGACGGGTCGT -3' & 5'- GAATTACTAACCGTACAACGCT -3' & 56 & 148 \\
\hline \multirow{2}{*}{ Myo G } & U: 5'- TATATTGACGGAGAAGTGTAGGTT-3' & 5'- CCСACATCTATAACAAAAATCACA -3' & 59 & 155 \\
\hline & M: 5'- GGTAGTTATATTGAGGGAGAAGC -3' & 5'- TCTAGAACGAAAATCGCGCTC -3' & 58 & 168 \\
\hline \multirow{2}{*}{$\operatorname{miR}-1$} & U: 5'- GATGTAATGGGAGATTTGG -3' & 5'- СССТCACACTAAACTAATAACCAC -3' & 56 & 158 \\
\hline & M: 5'- CGTAATGGGAGTTTCTGTT -3' & 5'- CGCCAATAATCTAATCAAGCT -3' & 59 & 153 \\
\hline \multirow{2}{*}{$\operatorname{miR}-133$} & U: 5'- GATGTAATGGGAGTTTTGG -3' & 5'- CСССТСАСАСТАТАСТАATAACC - $3^{\prime}$ & 54 & 158 \\
\hline & M: 5'- CGTAATGGGACTTTCGGTTC -3' & 5'- CTCCAACTAAACTAATAAGCGC - $3^{\prime}$ & 59 & 153 \\
\hline \multirow{2}{*}{ miR-206 } & U: 5'- TAGGAAGAGATAAAGTTTTAGGGT -3' & 5'-TTAACAACTCAACCTTCTACAATAATCA -3' & 55 & 222 \\
\hline & M: 5'- TAGGAAGAGTTACAGTTTTAGGGC -3' & 5'- CTCAACCTTCTAATATAATCGTT -3' & 59 & 215 \\
\hline
\end{tabular}

Note. U: Unmethylated; M: Methylated; PCR: Real-time polymerase chain reaction. 
as an n-fold. Our results indicated that DNMT1 gradually increased for 2 days, and then leveled down on days 3,4 , and 5 compared to day 0 in non-transfected C2C12 cells. As shown in Figure 1A, DNMT1 started decreasing on day 2 and had the lowest level on day 3 and then its level elevated on days 4 and 5. However, its level on days 2-5 was lower than that of the non-transfected cells. DNMT3A demonstrated nearly the same pattern as DNMT1, where the lowest level belonged to day 3 .

DNMT3A gradually increased for 2 days and then decreased on days 3, 4, and 5 as compared to day 0 (Figure 1A). DNMT3B increased on the first day and decreased on days 3,4 , and 5 of differentiation as compared to day 0 (Figure 1A). Moreover, these findings demonstrated that the average mRNA level of all three DNMTs (T1/3A/3B) in the non-transfected $\mathrm{C} 2 \mathrm{C} 12$ cells of the third day of differentiation were at the lowest expression compared to those of the cells on day 0 and then started leveling up on days 4 and 5. For Cdk9-transfected cells, the lowest level of DNMT1 was observed on day 5 in spite of nontransfected cells and its level started decreasing from day 2 until day 5 (Figure 2A). With a fluctuation, the overall level of DNMT3A increased all days compared to day 0 (Figure 2A). Moreover, the highest level of DNMT3B was observed on days 2 and 3 (Figure 2A). Based on the results (Figure $2 \mathrm{~B}$ ), there was a trend for the higher expression of the average levels of all three DNMTs (T1/3A/3B) in transfected $\mathrm{C} 2 \mathrm{C} 12$ cells during differentiation as compared to day 0 although differences were not significant $(P>0.05)$. Overall, the results (Figure 3$)$ demonstrated that the average expression levels of DNMTs noticeably increased $(\sim 2$-fold $)$ in transfected cells as compared to non-transfected control cells (non-transfected: 0.8-fold, transfected: 1.61 -fold $)(P<0.01)$.

\section{Global Methylation Analysis}

The total 5-mC content of the genomic DNA of nontransfected and transfected $\mathrm{C} 2 \mathrm{C} 12$ cells were quantified to examine if DNA methylation changes take place during $\mathrm{C} 2 \mathrm{C} 12$ differentiation. First, the levels of global DNA methylation in non-transfected $\mathrm{C} 2 \mathrm{C} 12$ cells were investigated in this regard. Our finding indicated that nearly, $1.143 \%, 1.07 \%, 1.06 \%, 1.05 \%, 1.14 \%$, and $1.08 \%$ of the cytosine residues were methylated at $0,1,2,3,4$, and 5 day(s) in the genome of non-transfected cells, respectively (Figure 4A). Approximately 1.2\%, 1.17\%, 1.07\%, 1.11\%, $1.14 \%$, and $1.13 \%$ of the cytosine residues were methylated at $0,1,2,3,4$, and 5 day(s) in the genome of the transfected cells, respectively (Figure 4B). Interestingly, the findings (Figure 4A-B) demonstrated that global DNA methylation levels remarkably reduced on the 2 nd day in both nontransfected and transfected $\mathrm{C} 2 \mathrm{C} 12$ cells $(P<0.05)$. Based on data in Figure $4 \mathrm{C}$, the levels of global methylation significantly increased in transfected cells in comparison with their respective non-transfected cells (increased: 1.044-fold, $P<0.05)$.

Association of CDK9 Overexpression With Cardiac Specific miRNAs and MRFs Promoter DNA Methylation The MSP on the specific sites was conducted to study the impact of $C d k 9$ overexpression on the promoter DNA methylation status of MyoD and Myogenin (MyoG), and
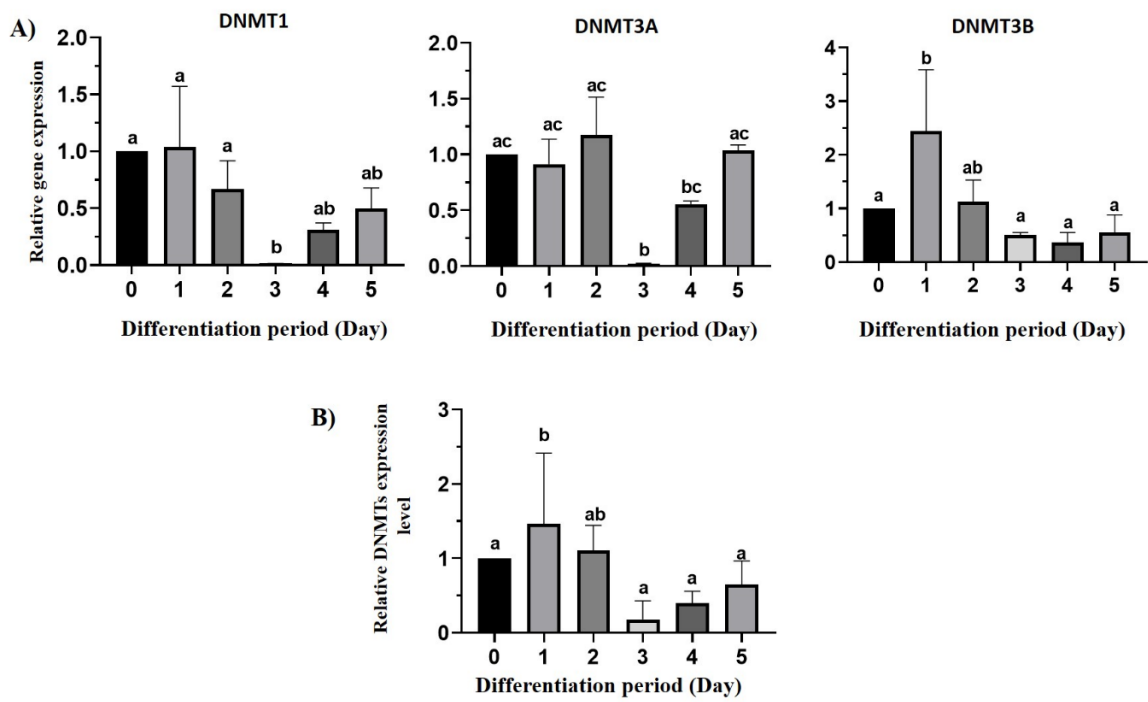

Figure 1. A. The Relative Expression of DNMT1, DNMT3A, and DNMT3B in C2C12 Non- transfected Cells During Differentiation Measured by RT-PCR and B. A Summary of the Change in the Average Expression of All 3 DNMTs (T1/3A/3B) in Non-transfected (Control) C2C12 Cells During Differentiation. Note. The expression of each gene was normalized to GAPDH. C2C12 cells at day 0 were considered as controls and the rate of the expression of the genes of interest in other groups was calculated as well. The data were presented as the mean \pm SD and each experiment was conducted in triplicate. The results were analyzed by one-way ANOVA and Tukey's post hoc tests. The significance level is considered as $P<0.05$. The bars marked with different letters are significantly different from other samples. C: Control (non-transfected); T: Transfect; RT-PCR: Real-time polymerase chain reaction; SD: Standard deviation. 

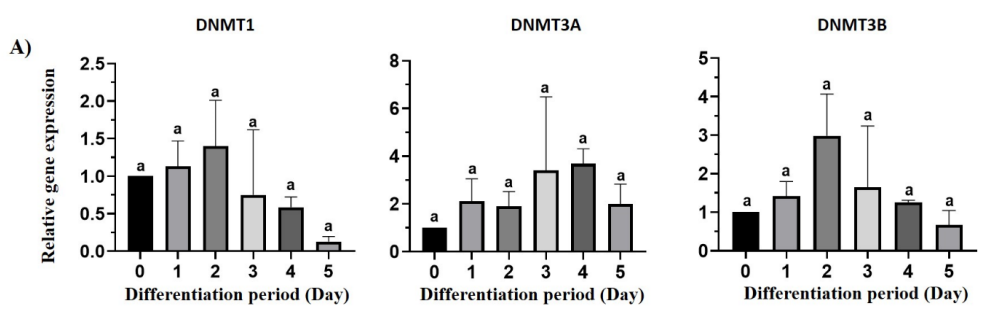

B)

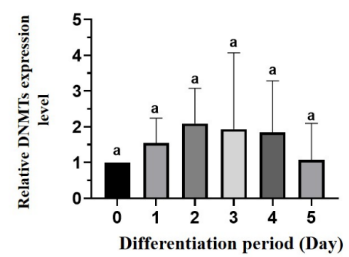

Figure 2. A. The Relative Expression of DNMT1, DNMT3A, and DNMT3B in Cdk9- transfected C2C12 Cells During Differentiation Measured by RT-PCR and B. A Summary of the Change in the Average Expression of all 3 DNMTs (T1/3A/3B) in Cdk9-transfected C2C12 Cells During Differentiation. Note. The expression of each gene was normalized to GAPDH. Cdk-9-transfected $\mathrm{C} 2 \mathrm{C} 12$ cells at day 0 were considered as controls and the rate of the expression of the genes of interest in other groups was computed as well. The data are presented as the mean \pm SD and each experiment was conducted in triplicate. The results were analyzed by one-way ANOVA and Tukey's post hoc tests. The significance level is considered as $P<0.05$. The bars marked with different letters significantly differ from other samples. C: Control (non-transfected); T: Transfect RT-PCR: Real-time polymerase chain reaction; SD: Standard deviation; ANOVA: Analysis of variance.

miR-1, miR-206, and miR-133 on days $0,1,2,3,4$, and 5 in C2C12 cells. The representative MSP of MyoD and Myogenin promoter methylation are shown in Figure 5. The MSP analysis indicated that the MyoD promoter was unmethylated in both non-transfected (control) and transfected C2C12 cells during differentiation although it was methylated only in control cells at day 5 (Figure 5). The hypermethylation of the Myogenin gene promoter was also found in both non-transfected and transfected cells at days 1-5 of differentiation (Figure 5). For miR-1, promoter methylation was only observed in transfected cells on day 4 (Figure 5). Moreover, the results confirmed that the promoter methylation of the miR-133 gene increased at days 4 and 5 in transfected cells as compared to control cells (Figure 5). Eventually, the results revealed a trend for miR-206 hypermethylation in both nontransfected and transfected cells, especially at days 4 and 5 of differentiation (Figure 5).

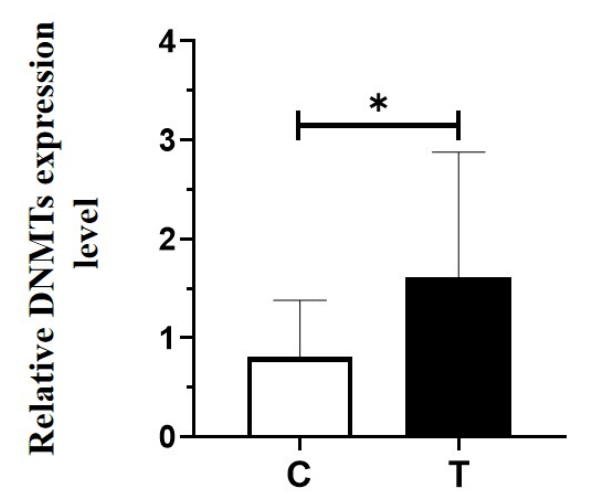

Figure 3. A Summary of the Change in the Average Gene Expression of All 3 DNMTs (T1/3A/3B) in Control and Cdk9-transfected C2C12 Cells During the Differentiation Period.

\section{Discussion}

This study highlights the significance of Cdk9 overexpression on regional and global DNA methylation in addition to DNMT gene expression changes in $\mathrm{C} 2 \mathrm{C} 12$ cells during differentiation. Moreover, regional and global DNA methylation changes and gene expression patterns were observed in Cdk9-transfected myoblast cells during differentiation. It was further found that Cdk9 overexpression is able to increase the average expression level of DNMTs and global DNA methylation, as well as modulating the regional DNA methylation of MRFs and myoRNAs in $\mathrm{C} 2 \mathrm{C} 12$ during differentiation. Muscle differentiation is a procedure with various steps organized by $C d k 9, M R F s$, and other epigenetic mechanisms including miRNAs and DNA methylation (22-28). Epigenetics define a regulatory mechanism of gene expression without changes in the underlying DNA sequences and play key roles in embryogenesis and differentiation (25). DNA methylation is a mechanism that donates a methyl group to the cytosine residue in CpG islands (5) by DNMT enzymes at the primary stage of embryogenesis $(5,26)$. Additionally, experimental studies indicated that the upregulation of specific DNMT isoforms leads to manifesting cell activation and differentiation of myogenic stem cells $(27,29,30)$.

A recent study has reported that $C d k 9$ is linked to gene silencing in mammals by chromatin remodeling and epigenetic modification (31). From the mechanistic viewpoint, it has been suggested that the $C d k 9$ induction pattern is roughly similar to DNMTs, and the synergy with DNMTs suggests $C d k 9$ as a novel epigenetic target for clinical developments $(31,32)$. However, the precise mechanism by which Cdk9 regulates DNMT gene expression, DNA global methylation, and MRFs and miRNAs promoter methylation in myoblast cells is not 
A)

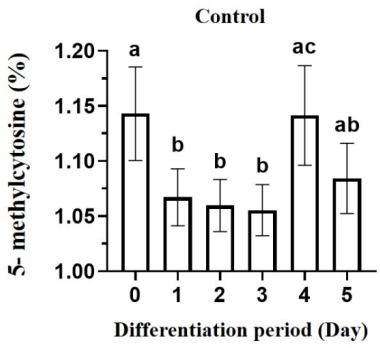

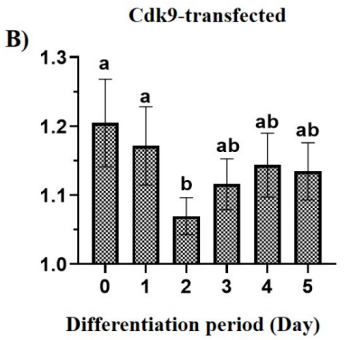

C)

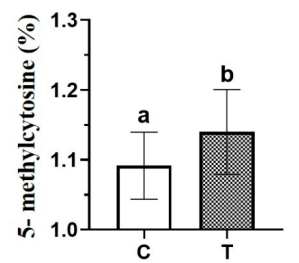

Figure 4. A. The Global DNA Methylation Levels in A. Control, B. Cdk9-transfected C2C12 Cells During the Differentiation Period, and C. A Summary of the Change in the Average Global Methylation Level in Control and Cdk9-transfected C2C12 Cells During the Differentiation Period. Note. The percentage of 5-mC in control and Cdk-9 transfected C2C12 cells was evaluated using ELISA assay. The mean values \pm SD of three experiments are provided. The results were analyzed by one-way ANOVA and Tukey's post hoc tests. The significance level is considered as $P<0.05$. C: Control (non-transfected); T: Transfected; SD: Standard deviation; ANOVA: Analysis of variance; ELISA: Enzyme-linked immunosorbent assay.

fully understood yet. Concerning Cdk9 regulatory roles, we examined the potential regulatory functions of $C d k 9$ overexpression on the entire genomic DNA methylation and regional promoter DNA methylation status of cardiac specific miRNA, MRFs as well as DNMT gene expression in myoblast $\mathrm{C} 2 \mathrm{C} 12$ cells.

To the best of our knowledge, this is the first time that $C d k 9$ is linked to DNA methylation during $\mathrm{C} 2 \mathrm{C} 12$ cell differentiation. This study is the first one to show that the Cdk9 overexpression is able to induce DNMT gene expression in Cdk9-transfected $\mathrm{C} 2 \mathrm{C} 12$ cells in comparison to non-transfected (control) $\mathrm{C} 2 \mathrm{C} 12$ cells during differentiation. Our results indicated that, in control C2C12 cells, only DNMT3B significantly increased in the first day of differentiation as compared to days $0,3,4$, and 5 (Figure 1A). The results (Figure 1A) also represented the lowest level of DNMT1 and
DNMT3A in non-transfected cells on day 3, as well as a significantly higher expression level of DNMT1 and DNMT3A in non-transfected C2C12 cells on the 0-2 days of differentiation as compared to day $3(P<0.05)$. Some researchers speculated that DNMT1 has responsibility for DNA methylation maintenance although DNMT (3A $\& 3 \mathrm{~B})$ contributes to de novo methylation during cell differentiation $(33,34)$. Overall, the highest and lowest levels of DNMTs (T1/3A/3B) and were observed on the first and the third to fifth days of $\mathrm{C} 2 \mathrm{C} 12$ differentiation in non-transfected cells (Figure 1B). Previous research also indicated that the expressions of DNMT1 and DNMT3A were diminished during the myogenic process (35). Moreover, the results regarding PCEP4/Cdk9 plasmid transfected cells demonstrated an increased expression level of DNMTs (T1/3A/3B) during differentiation (Figure $2 \mathrm{~B})$. Interestingly, our study showed that the expression

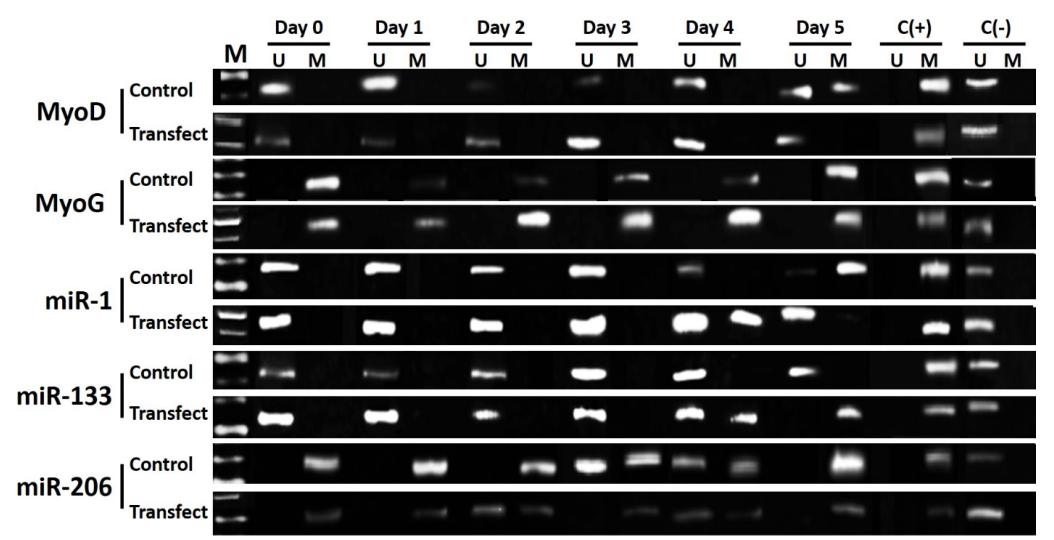

Figure 5. Representative Examples of MSP for the Promoter Methylation Analysis of MyoD, MyoG (Myogenin), miR-1, miR-133, and miR-206 in Control (nontransfected) and Cdk9-transfect C2C12 Cells. Note. U: Unmethylated; M: Methylated; M: 50 bp DNA size marker; C (+): Control positive; C (-): Control negative; MSP: Methylation-specific PCR. 
levels of DNMTs decrease in non-transfected cells while DNMTs expression levels increase in Cdk9-transfected cells during the differentiation process. Overall, although the control and transfected $\mathrm{C} 2 \mathrm{C} 12$ cells were cultured during the same conditions, the average transcription level of DNMT changes induced by Cdk9 was higher in transfected cells in comparison with non-transfected cells (wild: 0.8 -fold, transfected: 1.61-fold, $P<0.05$, Figure 3). This clearly indicates a different epigenetic modification during the differentiation in control and transfected $\mathrm{C} 2 \mathrm{C} 12$ cells. It has been suggested that $C d k 9$ is one of the main kinases required for epigenetic modifications and has a mechanistic function in epigenetic modifications for instance histone methylation (36). In addition, increasing evidence suggests that $C d k 9$ in combination with other transcription factors including CDK7 and RNA pol III regulates and increases gene body methylation, and therefore, increases the gene expression in cancer cells (37).

To investigate the global methylation level for nontransfected and Cdk9-transfected $\mathrm{C} 2 \mathrm{C} 12$ cells, the average level of the 5-mC content of the genomic DNA related to these cells was calculated during differentiation and it was found that the global DNA methylation level reduced in the same day $(2 \mathrm{~d})$ in both non-transfected and transfected $\mathrm{C} 2 \mathrm{C} 12$ cells $(P<0.05$, Figure 4A-B). Moreover, the findings (Figure $4 \mathrm{C}$ ) generally revealed that the Cdk9 overexpression is able to increase global DNA methylation in transfected $\mathrm{C} 2 \mathrm{C} 12$ cells in comparison to respective non-transfected (control) cells during differentiation $(P<0.05)$. Hence, an interesting probability raised in our research is that further active global DNA methylation in $\mathrm{C} 2 \mathrm{C} 12$ transfected cells can be explained by the increased DNMT expression by Cdk9 in the cells. DNA methylation is a key molecular mechanism required for the self-renewal and maintenance of stem cells during differentiation (38). Further, the $C d k 9$ regulatory role leads to increased efficiency of cardiac developments by the epigenetic programming of miRNA and MRFs including $M y o D$ and Myogenin $(8,16)$. In this study, it was reported that the $C d k 9$ overexpression is able to induce the promoter methylation of miR-1 and miR-133 in Cdk9- transfected C2C12 cells (Figure 5). It was also found the promoter methylation of miR-1 on day 4 and the promoter methylation of miR-133 increased on days 4 and 5 in transfected cells as compared to non-transfected cells (Figure 5). Some researchers speculated that the regulatory role of $C d k 9$ may be mediated by decreasing miR-133 gene expression and increasing miR1 and miR206 transcription in C2C12 myoblast cells $(15,17,39,40)$. Moreover, our results revealed that the MyoD promoter was unmethylated in both non-transfected and transfected $\mathrm{C} 2 \mathrm{C} 12$ cells during differentiation although it was methylated only in control non-transfected cells on day 5 (Figure 5). MyoD is the main regulator of the cardiac myogenic program and its expression is regulated by promoter epigenetic modifications during differentiation $(41,42)$. The increasing evidence suggests that the Cdk9 in combination with other key factors including Cyclin T2a plays a key role in the initiation of the myogenic procedure by stimulating $M y o D$ family transcription $(43,44)$. Moreover, our previous study showed that $C d k 9$ overexpression increased $M y o D$ gene expression in myoblast $\mathrm{C} 2 \mathrm{C} 12$ cells (15). Based on these results, Cdk9 probably increased $M y o D$ gene expression by reducing its promoter methylation. Furthermore, the hypermethylation of the Myogenin promoter was found in both cells on all days (Figure 5). The MRF transcripts including Myogenin are strongly critical for several stages of myogenesis (11). The precise mechanisms regulating DNA methylation throughout myogenesis remain unknown. However, extensive data indicate that the promoter demethylation of $M y o D$ and the Myogenin is necessary for proceeding the differentiation program (12). Our previous study indicated that continuous C $d k 9$ overexpression in transfected cells reduces Myogenin gene expression during differentiation (16). Our finding is in line with that of other studies, demonstrating that the Myogenin gene is present in a transcriptionally inhibited form in proliferating myoblasts (45). Moreover, the current findings revealed that Myogenin gene expression is reduced by the de novo methylation of its promoter region in terminally differentiated muscle cells, and other myogenic genes requiring terminal differentiation may be regulated by promoter DNA methylation $(46,47)$. Overall, a positive correlation was found between genomic global DNA methylation, DNMT gene expression, and regional methylation for cardiac-specific miomiRs including miR1 and miR-133 in Cdk9-transfected C2C12 cells.

\section{Conclusions}

In general, our results indicated that $C d k 9$ overexpression can promote the regional promoter DNA methylation of myomiRs including miR-1 and miR-133 in addition to increasing global DNA methylation and DNMT gene expression in myoblast $\mathrm{C} 2 \mathrm{C} 12$ cells. The finding offers novel insights into the mechanisms of epigenetic by which C $d k 9$ promotes epigenetic changes and modulates the global and regional DNA methylation profiling of myoblast cells during differentiation.

\begin{abstract}
Authors' Contribution
MSH and MMS conducted this study. LA, HK and VT performed the experiments. MSH, HG and MMS supervised the experiments. LA, SM, MMS and VT wrote the manuscript. MMS and MSH revised the manuscript. All of the authors assisted in writing the manuscript, discussed the results and commented on the manuscript.
\end{abstract}

\section{Conflict of Interests \\ None.}

\section{Ethical Issues}

There is no involvement of humans or animals in this study.

\section{Financial Support}

This work was supported by Tabriz University of Medical Sciences with grand number: IR.TBZMED.VCR.REC.1396.1188. 


\section{Acknowledgments}

The authors acknowledge the Molecular Medicine Research Center, Biomedicine Institute, Tabriz University of Medical Sciences, and Lorestan University of Medical Sciences, Khorramabad, Iran.

\section{References}

1. Laker RC, Ryall JG. DNA methylation in skeletal muscle stem cell specification, proliferation, and differentiation. Stem Cells Int. 2016;2016:5725927. doi:10.1155/2016/5725927

2. Ebrahimi V, Soleimanian A, Ebrahimi T, et al. Epigenetic modifications in gastric cancer: focus on DNA methylation. Gene. 2020;742:144577. doi:10.1016/j.gene.2020.144577

3. Christman JK. 5-Azacytidine and 5-aza-2'-deoxycytidine as inhibitors of DNA methylation: mechanistic studies and their implications for cancer therapy. Oncogene. 2002;21(35):54835495. doi:10.1038/sj.onc.1205699

4. Bogdanović O, Veenstra GJ. DNA methylation and methyl-CpG binding proteins: developmental requirements and function. Chromosoma. 2009;118(5):549-565. doi:10.1007/s00412-0090221-9

5. Smith ZD, Meissner A. DNA methylation: roles in mammalian development. Nat Rev Genet. 2013;14(3):204-220. doi:10.1038/ nrg3354

6. Deaton AM, Bird A. CpG islands and the regulation of transcription. Genes Dev. 2011;25(10):1010-1022. doi:10.1101/gad.2037511

7. Xie W, Barr CL, Kim A, et al. Base-resolution analyses of sequence and parent-of-origin dependent DNA methylation in the mouse genome. Cell. 2012;148(4):816-831. doi:10.1016/j. cell.2011.12.035

8. Ghanbarian H, Wagner N, Michiels JF, Cuzin F, Wagner KD, Rassoulzadegan M. Small RNA-directed epigenetic programming of embryonic stem cell cardiac differentiation. Sci Rep. 2017;7:41799. doi:10.1038/srep41799

9. Ghanbarian H, Wagner N, Polo B, et al. Dnmt2/Trdmt1 as mediator of RNA polymerase II transcriptional activity in cardiac growth. PLoS One. 2016;11(6):e0156953. doi:10.1371/journal. pone.0156953

10. Abkhooie L, Sarabi MM, Kahroba H, et al. Potential roles of MyomiRs in cardiac development and related diseases. Curr Cardiol Rev. 2020. doi:10.2174/1573403×16999201124201021

11. Knight JD, Kothary R. The myogenic kinome: protein kinases critical to mammalian skeletal myogenesis. Skelet Muscle. 2011;1:29. doi:10.1186/2044-5040-1-29

12. Perdiguero E, Sousa-Victor P, Ballestar E, Muñoz-Cánoves P. Epigenetic regulation of myogenesis. Epigenetics. 2009;4(8):541550. doi:10.4161/epi.4.8.10258

13. Sano $\mathrm{M}$, Abdellatif $\mathrm{M}, \mathrm{Oh} \mathrm{H}$, et al. Activation and function of cyclin T-Cdk9 (positive transcription elongation factor-b) in cardiac muscle-cell hypertrophy. Nat Med. 2002;8(11):13101317. doi:10.1038/nm778

14. Ghanbarian H, Grandjean V, Cuzin F, Rassoulzadegan M. A network of regulations by small non-coding RNAs: the P-TEFb kinase in development and pathology. Front Genet. 2011;2:95. doi:10.3389/fgene.2011.00095

15. Tarhriz V, Wagner KD, Masoumi Z, Molavi O, Hejazi MS, Ghanbarian H. CDK9 regulates apoptosis of myoblast cells by modulation of microRNA-1 expression. J Cell Biochem. 2018;119(1):547-554. doi:10.1002/jcb.26213

16. Tarhriz V, Eyvazi S, Musavi M, et al. Transient induction of Cdk9 in the early stage of differentiation is critical for myogenesis. J Cell Biochem. 2019;120(11):18854-18861. doi:10.1002/jcb.29204

17. Simone $C$, Stiegler $\mathrm{P}$, Bagella $\mathrm{L}$, et al. Activation of MyoD-dependent transcription by cdk9/cyclin T2. Oncogene. 2002;21(26):41374148. doi:10.1038/sj.onc. 1205493

18. Livak KJ, Schmittgen TD. Analysis of relative gene expression data using real-time quantitative PCR and the 2(-Delta Delta C(T)) Method. Methods. 2001;25(4):402-408. doi:10.1006/ meth.2001.1262

19. Moradi Sarabi M, Zahedi SA, Pajouhi N, et al. The effects of dietary polyunsaturated fatty acids on miR-126 promoter DNA methylation status and VEGF protein expression in the colorectal cancer cells. Genes Nutr. 2018;13:32. doi:10.1186/s12263-0180623-5

20. Mokarram P, Shakiba-Jam F, Kavousipour S, Moradi Sarabi M Seghatoleslam A. Promoter methylation status of two novel human genes, UBE2Q1 and UBE2Q2, in colorectal cancer: a new finding in Iranian patients. Asian Pac J Cancer Prev. 2015;16(18):82478252. doi:10.7314/apjcp.2015.16.18.8247

21. Moradi Sarabi M, Naghibalhossaini F. Association of DNA methyltransferases expression with global and gene-specific DNA methylation in colorectal cancer cells. Cell Biochem Funct. 2015:33(7):427-433. doi:10.1002/cbf.3126

22. Segalés J, Perdiguero E, Muñoz-Cánoves P. Epigenetic control of adult skeletal muscle stem cell functions. FEBS J. 2015;282(9):15711588. doi:10.1111/febs.13065

23. van Rooij E, Liu N, Olson EN. MicroRNAs flex their muscles. Trends Genet. 2008;24(4):159-166. doi:10.1016/j.tig.2008.01.007

24. Klose RJ, Bird AP. Genomic DNA methylation: the mark and its mediators. Trends Biochem Sci. 2006;31(2):89-97. doi:10.1016/j. tibs.2005.12.008

25. Pepin ME, Ha CM, Crossman DK, et al. Genome-wide DNA methylation encodes cardiac transcriptional reprogramming in human ischemic heart failure. Lab Invest. 2019;99(3):371-386. doi:10.1038/s41374-018-0104-x

26. Okano M, Bell DW, Haber DA, Li E. DNA methyltransferases Dnmt3a and Dnmt3b are essential for de novo methylation and mammalian development. Cell. 1999;99(3):247-257. doi:10.1016/ s0092-8674(00)81656-6

27. Liu Y, Sun L, Jost JP. In differentiating mouse myoblasts DNA methyltransferase is posttranscriptionally and posttranslationally regulated. Nucleic Acids Res. 1996;24(14):2718-2722. doi:10.1093/nar/24.14.2718

28. Eyvazi S, Hejazi MS, Kahroba H, Abasi M, Zamiri RE, Tarhriz V. CDK9 as an appealing target for therapeutic interventions. Curr Drug Targets. 2019;20(4):453-464. doi:10.2174/1389450119666 181026152221

29. Duymich CE, Charlet J, Yang X, Jones PA, Liang G. DNMT3B isoforms without catalytic activity stimulate gene body methylation as accessory proteins in somatic cells. Nat Commun. 2016;7:11453. doi:10.1038/ncomms11453

30. Liao J, Karnik R, Gu H, et al. Targeted disruption of DNMT1, DNMT3A and DNMT3B in human embryonic stem cells. Nat Genet. 2015;47(5):469-478. doi:10.1038/ng.3258

31. Zhang $\mathrm{H}$, Pandey $\mathrm{S}$, Travers $\mathrm{M}$, et al. Targeting CDK9 reactivates epigenetically silenced genes in cancer. Cell. 2018;175(5):12441258.e1226. doi:10.1016/j.cell.2018.09.051

32. Krystof V, Baumli S, Fürst R. Perspective of cyclin-dependent kinase 9 (CDK9) as a drug target. Curr Pharm Des. 2012;18(20):28832890. doi:10.2174/138161212800672750

33. Jeltsch A, Jurkowska RZ. New concepts in DNA methylation. Trends Biochem Sci. 2014;39(7):310-318. doi:10.1016/j. tibs.2014.05.002

34. Trowbridge JJ, Snow JW, Kim J, Orkin SH. DNA methyltransferase 1 is essential for and uniquely regulates hematopoietic stem and progenitor cells. Cell Stem Cell. 2009;5(4):442-449. doi:10.1016/j. stem.2009.08.016

35. Trowbridge JJ, Snow JW, Kim J, Orkin SH. DNA methyltransferase 1 is essential for and uniquely regulates hematopoietic stem and progenitor cells. Cell Stem Cell. 2009;5(4):442-449. doi:10.1016/j. stem.2009.08.016

36. Eissenberg JC, Shilatifard A, Dorokhov N, Michener DE. Cdk9 is an essential kinase in Drosophila that is required for heat shock gene expression, histone methylation and elongation factor recruitment. Mol Genet Genomics. 2007;277(2):101-114. doi:10.1007/ s00438-006-0164-2

37. Wong $\mathrm{CH}$, $\mathrm{Li} \mathrm{CH}, \mathrm{He}$ Q, Tong JHM, To KF, Chen Y. The Establishment of CDK9/ RNA PollI/H3K4me3/DNA Methylation Feedback Promotes HOTAIR Expression by RNA Elongation Enhancement in 
Cancer. bioRxiv. 2019:812776. doi:10.1101/812776

38. Berdasco M, Esteller M. DNA methylation in stem cell renewal and multipotency. Stem Cell Res Ther. 2011;2(5):42. doi:10.1186/ scrt83

39. Rosenberg Ml, Georges SA, Asawachaicharn A, Analau E, Tapscott SJ. MyoD inhibits Fstl1 and Utrn expression by inducing transcription of miR-206. J Cell Biol. 2006;175(1):77-85. doi:10.1083/jcb.200603039

40. Chen JF, Mandel EM, Thomson JM, et al. The role of microRNA-1 and microRNA-133 in skeletal muscle proliferation and differentiation. Nat Genet. 2006;38(2):228-233. doi:10.1038/ ng1725

41. Blum R, Dynlacht BD. The role of MyoD1 and histone modifications in the activation of muscle enhancers. Epigenetics. 2013;8(8):778784. doi:10.4161/epi.25441

42. Wardle FC. Master control: transcriptional regulation of mammalian Myod. J Muscle Res Cell Motil. 2019;40(2):211-226. doi:10.1007/s10974-019-09538-6

43. Giacinti C, Bagella L, Puri PL, Giordano A, Simone C. MyoD recruits the cdk9/cyclin T2 complex on myogenic-genes regulatory regions. J Cell Physiol. 2006;206(3):807-813. doi:10.1002/ jcp.20523

44. Weintraub $H$, Davis $R$, Tapscott $S$, et al. The myoD gene family: nodal point during specification of the muscle cell lineage. Science. 1991;251(4995):761-766. doi:10.1126/science.1846704

45. Fuso A, Ferraguti G, Grandoni $F$, et al. Early demethylation of non- $\mathrm{CpG}, \mathrm{CpC}$-rich, elements in the myogenin 5 '-flanking region: a priming effect on the spreading of active demethylation. Cell Cycle. 2010;9(19):3965-3976. doi:10.4161/cc.9.19.13193

46. Miyata K, Miyata T, Nakabayashi K, et al. DNA methylation analysis of human myoblasts during in vitro myogenic differentiation: de novo methylation of promoters of muscle-related genes and its involvement in transcriptional down-regulation. Hum Mol Genet. 2015;24(2):410-423. doi:10.1093/hmg/ddu457

47. Oikawa Y, Omori R, Nishii T, Ishida Y, Kawaichi M, Matsuda E. The methyl-CpG-binding protein CIBZ suppresses myogenic differentiation by directly inhibiting myogenin expression. Cell Res. 2011;21(11):1578-1590. doi:10.1038/cr.2011.90

Copyright $\odot 2022$ The Author(s); This is an open-access article distributed under the terms of the Creative Commons Attribution License (http://creativecommons.org/licenses/by/4.0), which permits unrestricted use, distribution, and reproduction in any medium, provided the original work is properly cited. 